\title{
Accurate Cryo-EM Characterizations of Polypeptoid Vesicles
}

\author{
Xi Jiang ${ }^{1}$, Jing Sun ${ }^{2,5}$, Ronald N. Zuckermann ${ }^{2}$, Nitash P. Balsara ${ }^{1,4}$ and Kenneth H. Downing ${ }^{3}$ \\ ${ }^{1 .}$ Materials Science Division, ${ }^{2 .}$ Molecular Foundry, ${ }^{3 .}$ Molecular Biophysics and Integrated Bioimaging \\ Division, Lawrence Berkeley National Lab, Berkeley CA, 94720 \\ 4. Department of Chemical and Biomolecular Engineering, University of California, Berkeley, CA \\ 94720.
}

5. Qingdao University of Science and Technology, Qingdao, China 266042

Vesicles are spherical bubbles that enclose aqueous solvent with a molecularly thin membrane. The membranes generally comprise self-assembled amphiphilic molecules with a hydrophilic-hydrophobichydrophilic character. They can be either naturally formed or artificially produced, such as liposomes composed of phospholipid and polymersomes formed by various amphiphilic block copolymers.

Peptoids are a highly tunable polymer family that allows precise control of the monomer sequence. Peptoids are based on an N-substituted glycine backbone, and can have a variety of chemically diverse side chains, making them close structural relatives of natural polypeptides. Sequence-defined peptoid polymers can be synthesized from simple precursors by an iterative solid-phase method, enabling the efficient synthesis of polymers with precise control over chain length (PDI $<1.0003$ ) and copolymer composition [1]. In this study, a series of sequence-defined amphiphilic polypeptoid block copolymers (BCPs) that contain a phosphonated block and an amorphous hydrophobic block: poly- $N$-(2-ethyl) hexylglycine-block-poly- $N$-phosphonomethylglycine (pNeh- $b$-pNpm) were synthesized with exactly same chain length [2]. Polypeptoid vesicles were produced by dissolving these amphiphilic polypeptoid BCPs in THF/water 50/50 solvent as shown in Figure 1 (a). Cryo-EM imaging of frozen hydrated vesicles was used to determine the morphology, size distribution, and membrane thickness of the vesicles.

It is of great importance to accurately characterize the structure of these vesicle, in particular their thickness, since membrane thickness is an important factor that affects many activities and functions. In most of previous studies on vesicles, the membrane thickness was measured directly from either negatively stained or cryo-EM micrographs $[3,4]$. It is difficult to precisely measure membrane thickness for negatively stained vesicles, especially the thin hydrophilic layer, because of the artifacts induced by chemicals. On the other hand, the measurement in cryo-EM micrographs are affected by low signal to noise ratio (SNR) as shown in Figure 1(b), due to the radiation sensitivity of the membranes. Cryo-electron tomography can be used to increase SNR by $3 \mathrm{D}$ reconstruction but the resolution is limited to the nanometer scale. In order to accurately measure the thickness of individual hydrophilic and hydrophobic layers in the membranes, the signal to noise ratio in cryo-EM micrographs was significantly enhanced by using single particle analysis in this study. The membranes in vesicles are homogeneous in terms of bilayer structure with similar thickness. Thus, hundreds of small micrograph segments containing membranes with similar curvature were extracted from vesicle micrographs. The averaged image of the membrane indicates sufficiently enhanced signal to noise ratio to support the accurate measurement for both the thin hydrophilic layer and hydrophobic layer after 2D classification, and averaging as Figure 1(c) shows. While the SNR in the averaged image is tremendously increased, the Fresnel fringe suggests a strong effect of defocus on the measurements. One of the most important properties of the polypeptoids in this study is the precisely controlled chain length with varying 
hydrophilic/hydrophobic segment content. We want to evaluate the relative contributions of these regions to the images. EM image simulation of polypeptoid vesicles with various defocus values allows us to evaluate the effect of defocus on the measurements in averaged images. Eventually, the thickness of both thin hydrophilic layers and hydrophobic layers can be accurately measured with sub-nm resolution and the structure of polypeptoid membranes can be revealed on the molecular level.

[1] E.J. Robertson, A Battigelli, C Proulx et al, Acc. Chem. Res, 49 (2016), p379.

[2] J Sun, X Jiang, A Siegmund, Macromolecules, 49 (2016), p3083.

[3] G Battaglia, A. J. Ryan, J. Am. Chem. Soc, 127 (2005), p875

[4] D.E. Discher, A Eisenberg, Science, 297 (2002), p967

[5] Funding for this work was provided by the Soft Matter Electron Microscopy Program, supported by the Office of Science, Office of Basic Energy Science, U.S. Department of Energy, under contract no. DE-AC02-05CH11231.

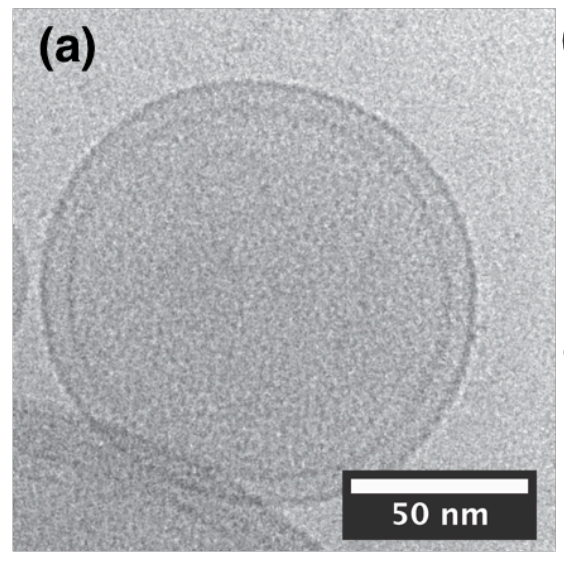

(b)
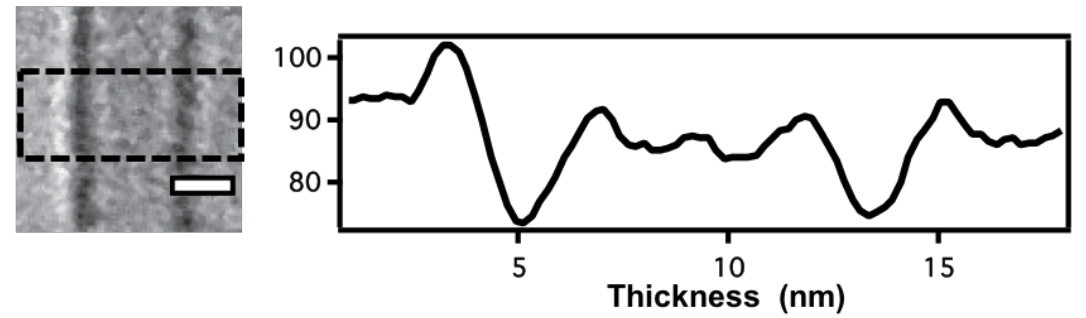

(c)
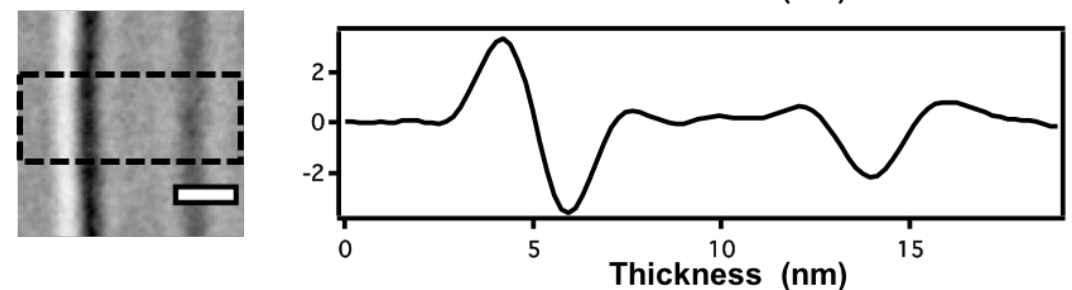

Figure 1. Cryo-EM micrograph of Polypeptoid vesicle (a). Initial membrane image and corresponding thickness profile of membrane (b). The area boxed with the dash line indicates the regions for measurement. Averaged membrane image and corresponding thickness profile of membrane (c). Scale bar in (b) and (c) is $5 \mathrm{~nm}$. 